INPLASY

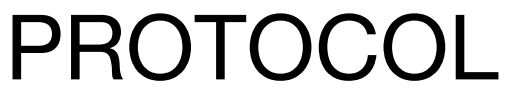

To cite: Kim et al.

Complementary and

Integrative Health Practices for

Individuals with Traumatic

Brain Injury: A Scoping

Review. Inplasy protocol

202160071. doi:

10.37766/inplasy2021.6.0071

Received: 21 June 2021

Published: 21 June 2021

Corresponding author:

Sonya kim

sonya.kim@nyulangone.org

Author Affiliation:

New York University Grossman

School of Medicine.

Support: ACRM.

Review Stage at time of this

submission: Data analysis

complete, not yet published.

Conflicts of interest:

None declared.

\section{Complementary and Integrative Health Practices for Individuals with Traumatic Brain Injury: A Scoping Review}

Kim, S1; Mortera, MH2; Reed, WR3; Chin, B4; Wen, PS5; Lundgren, $\mathrm{K}^{6}$; Sasson, N7; Thompson, $\mathrm{K}^{8}$; Wright, $\mathrm{S}^{9}$; Joseph, J10; Krishnan, S11; Heyn, PC ${ }^{12}$.

Review question / Objective: This scoping review is significant in three ways: it identifies which types of research have been done over the most recent decades; evaluates the evidence for the varied CIM treatments for TBI; and utilizes this scoping review as a tool for improving future CIM research, which leads to further CIM acceptance and encourages increased utilization of science-driven, effective CIM in TBI rehabilitation.

Condition being studied: Traumatic brain injury.

Information sources: The literature searches were performed using the following bibliographic databases and interfaces: Medline (OvidSP), PubMed (NLM), Embase (Embase.com), CINAHL (EBSCO), Psyclnfo (OvidSP), and the Cochrane Library (Wiley). Searches included publications dating from 1992 to 2020 for each database. To supplement these formal database searches, additional citations were found in Google Scholar and the National Guidelines Clearinghouse.

INPLASY registration number: This protocol was registered with the International Platform of Registered Systematic Review and Meta-Analysis Protocols (INPLASY) on 21 June 2021 and was last updated on 21 June 2021 (registration number INPLASY202160071).

\section{INTRODUCTION}

Review question / Objective: This scoping review is significant in three ways: it identifies which types of research have been done over the most recent decades; evaluates the evidence for the varied CIM treatments for TBI; and utilizes this scoping review as a tool for improving future CIM research, which leads to further CIM 
acceptance and encourages increased utilization of science-driven, effective CIM in TBI rehabilitation.

Rationale: In recent years, complementary and integrative medicine (CIM) has been increasingly incorporated into more traditional approaches to traumatic brain injury (TBI) rehabilitation, yet effectiveness research remains largely unexamined. Since the 1990s, evidence in support of the use of CIM for TBI rehabilitation has increased. Yet, there has never been a scoping review of this magnitude to evaluate the growing literature.

Condition being studied: Traumatic brain injury.

\section{METHODS}

Search strategy: The literature searches were performed using the following bibliographic databases and interfaces: Medline (OvidSP), PubMed (NLM), Embase (Embase.com), CINAHL (EBSCO), Psyclnfo (OvidSP), and the Cochrane Library (Wiley). Searches included publications dating from 1992 to 2020 for each database. To supplement these formal database searches, additional citations were found in Google Scholar and the National Guidelines Clearinghouse. Traumatic brain injury and CIM were searched using multiple text key words and subject headings, such as "TBI," "head injury," "concussion," "brain injuries," and CIM terms such as "acupuncture", "Ayurveda", "chelation therapy", "chiropractic/osteopathic manipulation", "massage," "biofeedback", "meditation", "yoga".

Participant or population: Individuals with TBI at any age, all outcomes, and any TBI severity.

\section{Intervention: NA}

\section{Comparator: NA.}

Study designs to be included: Inclusion criteria were any quantitative study design published from 1992 to 2020 that investigated the efficacy of CIM interventions.

Eligibility criteria: Inclusion criteria were any quantitative study design published from 1992 to 2020 that investigated the efficacy of CIM interventions for individuals with TBI at any age, all outcomes, and any TBI severity. Studies reported at least one of the following, or a related intervention term. These interventions were derived from the 2012 National Health Interview Survey (NHIS) conducted by the National Center for Complementary and Integrative Health (NCCIH) on the use of 18 nonconventional complementary and integrative health care practices by adults and children. 1. Acupuncture 2. Ayurveda 3. Biofeedback 4. Chelation therapy 5. Chiropractic or osteopathic manipulation 6. Craniosacral therapy 7. Energy healing therapy 8. Hypnosis 9. Massage 10. Naturopathy 11. Traditional healers 12. Movement therapies (Pilates/Trager psychophysical integration/ Feldenkrais), 13. Herbal and non-vitamin supplements 14. Vitamins and minerals 15 . Homeopathy 16. Special diets 17. Yoga/tai and chi/qi gong 18. Relaxation techniques, defined as meditation, guided imagery, and progressive relaxation). This review included CIM from the above: (1) acupuncture, (2) biofeedback and neurofeedback (3), chelation therapy, (4) chiropractic or osteopathic manipulation, (5) energy healing therapy, (6) massage, (7) naturopathy, (8) traditional healers, (9) movement therapies (Pilates/Trager psychophysical integration/ Feldenkrais), (10) yoga/tai and chi/qi gong, (11) and relaxation techniques, defined as meditation, guided imagery, and progressive relaxation.

Information sources: The literature searches were performed using the following bibliographic databases and interfaces: Medline (OvidSP), PubMed (NLM), Embase (Embase.com), CINAHL (EBSCO), Psyclnfo (OvidSP), and the Cochrane Library (Wiley). Searches included publications dating from 1992 to 2020 for each database. To supplement these formal database searches, additional 
citations were found in Google Scholar and the National Guidelines Clearinghouse.

Main outcome(s): To identify gaps in the published literature and evaluate the evidence for and study characteristics of the primary research on CIM and TBI.

Data management: A data extraction spreadsheet was developed to confirm relevance and to record study characteristics. This spreadsheet was piloted and revised as needed by the review team before implementation. Studies not meeting inclusion criteria were excluded.

Quality assessment / Risk of bias analysis: The American Academy of Neurology (AAN) guideline was used to assign classification of levels of evidence to the included studies with Class I as the most rigorous randomized controlled trial (RCT) and Class IV as the least rigorous, including consensus or expert opinion.

Strategy of data synthesis: The data were compiled into a single spreadsheet using Microsoft Excel 2019 (Microsoft Corporation, Redmond, WA) for validation, coding, and analyses. Descriptive statistics were calculated to summarize the data. Percentile values were rounded to the nearest integer.

Subgroup analysis: Subgroup analyses was performed according to CIM modality, characteristics of the TBI, e.g., severity, time-post injury, and available demographics

Sensitivity analysis: NA.

Language: English.

Country(ies) involved: USA.

Keywords: Traumatic brain injury; complementary integrative health. practices.

Dissemination plans: Data will be published in a peer-reviewed journal as well as presented at the American Congress of Rehabilitation Medicine.

Contributions of each author:

Author 1 - Sonya Kim - conceptualization, data collection, creation of the database, data management data analysis, writing.

Email: sonya.kim@nyulangone.org

Author 2 - Marianne H. Mortera conceptualization, data collection, writing.

Email: mariannehmortera@gmail.com

Author 3 - William R. Reed conceptualization, data collection, writing.

Email: wreed@uab.edu

Author 4 - Bridget Chin - conceptualization, data collection, writing.

Email: bridgetchin@gmail.com

Author 5 - Pey-Shan Wen conceptualization, data collection, writing.

Email: pwen@gsu.edu

Author 6 - Kristine Lundgren conceptualization, data collection, writing.

Email: k_lundgr@uncg.edu

Author 7 - Nicole Sasson conceptualization, data collection, writing.

Email: Nicole.Sasson@va.gov

Author 8 - Karla L. Thompson conceptualization, data collection, writing.

Email: karla_thompson@med.unc.edu

Author 9 - Sarah Wright - developed the search strategies and covidence citations.

Email: wrightst@med.unc.edu

Author 10 - Justin Joseph - data collection, writing.

Email: justin.k.joseph@emory.edu

Author 11 - Shilpa Krishnan - data collection, writing.

Email: shilpa.krishnan@emory.edu

Author 12 - Patricia C. Heyn conceptualization, writing.

Email: PATRICIA.HEYN@CUANSCHUTZ.EDU 